\title{
The Influence of the Industrial Processing on the Degradation of Poly(hidroxybutyrate) - PHB
}

\author{
Wagner Maurício Pachekoski*, Carla Dalmolin ${ }^{\text {a }}$,José Augusto Marcondes Agnelli ${ }^{\mathrm{b}}$
}

\author{
aÁrea de Materiais, SENAI Cimatec, Av. Orlando Gomes, 1845, CEP 41650-010, Salvador, BA, Brasil \\ ${ }^{\mathrm{b}}$ Departamento de Engenharia de Materiais, Universidade Federal de São Carlos - UFSCar, \\ Rod. Washington Luis, Km 235, CEP 13565-905, São Carlos, SP, Brasil
}

Received: May 28, 2012; Revised: October 6, 2012

\begin{abstract}
PHB was characterized after different industrial processes by Differential Scanning Calorimetry (DSC), Fourier Transform Infrared Spectroscopy (FTIR), Melt Flow Index (MFI), Complex Dielectric Relaxation (CDR) and Size Exclusion Chromatography (SEC). Some properties of PHB were investigated before and after processing, in order to understand how temperature and other extrusion or injection conditions affect the polymer degradation. All the processed samples showed an increasing in the melt flow index, a decreasing of the dynamic crystallization temperature, and a reduction in the molar mass, suggesting some degradation. The molar mass reduction after processing, predicted when only thermal degradation is considered, was calculated in function of the kinetic parameters, such as constant thermal degradation and residence time during the industrial processing. It was found that the real molar mass reduction was higher than the theoretical value, indicating an important contribution of the shearing of polymeric chains during processing in the PHB degradation.
\end{abstract}

Keywords: poly(hydroxybutyrate), PHB, biodegradable polymer, degradation

\section{Introduction}

Poly(hydroxybutyrate) - PHB - is a biodegradable polymer that can be synthesized by bacterial fermentation using renewable raw materials such as sugarcane ${ }^{1}$. PHB has high crystallinity, hydrophobicity, is a $100 \%$ biodegradable $^{2}$ and, like most thermoplastics, can be processed by extrusion, injection, and melt press molding ${ }^{3}$. However, PHB has a limited stability at high temperatures required for melting processes $^{4,5}$ and undergoes thermal degradation, which affects its physical and mechanical properties, impairing industrial application ${ }^{6}$.

It has been shown that the thermal degradation of PHB is caused almost exclusively by a random scission on the ester bond at high temperatures (above $200^{\circ} \mathrm{C}$ ). This degradation is stereoselective, by a non-radical cis-elimination mechanism and shearing of the polymeric chain, leading to the reduction of the molar mass of the polymer and the formation of crotonic acid, with an activation energy of $235 \mathrm{~kJ}^{7,8}$. It was found that, after processing, the viscosity of PHB decreases due to degradation by random leakage of polymer chains. In extrusion, the molecular weight is proportional to the residence time, although no relation was found between the polidispersivity and the same parameter ${ }^{9}$. Further studies about mechanical properties of extruded PHB showed that the average number of molecular weight decreases with the increasing of temperature and screw speed, along with a significantly drop observed for

*e-mail: wagner.pachekoski@ fieb.org.br the elongation and stress at break values, while elasticity modulus was not affected ${ }^{10}$.

On the other hand, the biodegradation rate of PHB increases when the polymer is processed at higher temperatures, which is explained by the decreasing of the polymer crystallinity, which facilitates the capability of the extra-cellular enzymes responsible for $\mathrm{PBH}$ degradation to attack its chains ${ }^{11}$.

More recently, some efforts to overcome the poor processing window of $\mathrm{PHB}$ are directed to the preparation of copolymers with, for example, poly-(3-hydroxyvalerate $(\mathrm{HV})^{12}$ or poly-(3-hydroxyhexanoate) $(\mathrm{HH})^{13}$. Thermal, rheological and mechanical properties of these copolymers were investigated, showing the improvement on thermal stability for some compositions, although they are still unstable at temperatures higher than $160{ }^{\circ} \mathrm{C}$. The use of polymeric additives such as carboxyl-terminated butadiene acrylonitrile rubber (CTBN) and biocompatible polyvinylpyrrolidone (PVP) were also studied, in order to achieve thermal stability and controlled crystallization of $\mathrm{PBH}^{14}$. The results indicate that crystallization rate, crystallinity, and crystal size were significantly affected, because the additives affected the degraded reaction, highlighting the correlation between crystallization and the PHB thermal degradation.

Most studies about PHB degradation are concerned only about thermal degradation, and were carried out 
using samples prepared in laboratory conditions, such as casting, compression using molding sheets, or even the PHB powder without any processing methodology. To be suitable for common industrial applications, however, PHB should be processed in large scale, mostly by melt processing techniques such as extrusion and/or injection. In this case, the polymeric chains are submitted not only to high temperatures (above $170{ }^{\circ} \mathrm{C}$ for $\mathrm{PHB}$ ), but also to the shearing tension. When this tension is excessive, it may lead to a scission on the polymeric chain, causing reduction in the molar mass and characterizing a further degradation.

Based on these findings, the present work aims to characterize the degradation caused by heating and/or shearing resulting from the melt processing of PHB. Techniques such as Differential Scanning Calorimetry, Melt Flow Index, Complex Dielectric Relaxation, Infrared Spectroscopy and Size Exclusion Chromatography were used to study changes of molar mass and chemical structure in samples of PHB after common industrial processing, such as extrusion, or extrusion followed by injection.

\section{Experimental}

The poly(hydroxybutyrate $)-\mathrm{PHB}-\left(\overline{M_{w}}\right.$ of 534,000 g. $\mathrm{mol}^{-1}$ ) was manufactured at PHB Industrial S/A by the FE 145 fermentation process. Table 1 lists extrusion and injection parameters which represent an optimized situation of processing with minimum thermal degradation. PHB is a white powder that was processed in an Imacom modular twin-screw co-rotational extruder $\mathrm{L} / \mathrm{D}=30$, in order to produce PHB pellets. For studies about degradation after extrusion and injection, these pellets were then processed in an Arburg 270V-300-120 Allrounder injection machine, with a mold for injecting tensile (ASTM 638) and impact (ASTM 256) test specimen. Residence time of PHB during extrusion and injection was measured as 30 seconds for each processing step.

Thermal studies were made by Melt Flow Index (MFI) and Differential Scanning Calorimetry (DSC). MFI measurements were taken according to the condition foreseen for polyethylene under the ASTM 1238 standard, using a DSM MI-1 plastometer at $190{ }^{\circ} \mathrm{C}$, under a load of 2,160 g and cut-off time of 10 seconds. A TA Instruments DSC Q100 calorimeter was used for the DSC characterization in the range of -25 to $200{ }^{\circ} \mathrm{C}$ at a heating rate of $20^{\circ} \mathrm{C} / \mathrm{min}$ and a cooling rate of $80^{\circ} \mathrm{C} / \mathrm{min}$ under $\mathrm{N}_{2}$ atmosphere. The crystallinity $\left(X_{c}\right)$ was calculated from the melting peak in conventional DSC curves as follows:

$$
X_{c}=\frac{\Delta H_{\exp }}{\Delta H_{100 \%}}
$$

Where $\Delta H_{\text {exp }}$ is the area of the melting peak obtained by the experimental curves, and $\Delta H_{100 \%}$ is the enthalpy for $100 \%$

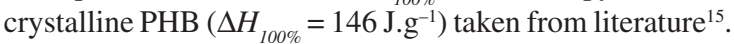

Changes on polymers structure after processing were investigated by Fourrier-Transformed Infrared Spectroscopy (FT-IR), Size Exclusion Chromatography (SEC), and Complex Dielectric Relaxation (CDR). IR spectra were recorded by a Perkin Elmer Spectrum 1000 FTIR spectrometer, in the region $4000-400 \mathrm{~cm}^{-1}$. A Tosoh chromatograph with a refraction index detector system was used to characterize the molar mass of PHB before and after processing by SEC. The solvent in the mobile phase was chloroform and the analytical column $(300 \mathrm{~mm} \times 7.8 \mathrm{~mm})$ used was Water Styragel HR $(7.8 \times 300 \mathrm{~mm})$. Dielectric relaxation experiments (CDR) were performed using a Novocontrol GmbH Concept 12 System coupled to a Solartron SI 1260 Impedance analyzer and a measuring cell. PHB films of $\approx 30 \mu \mathrm{m}$ in thickness were prepared by dissolution in chloroform and subsequent casting at room temperature. Circular Au electrodes $(3 \mathrm{~cm}$ in diameter) were deposited onto the film surfaces by sputtering. Measurements on the frequency domain of the real and imaginary parts of the dielectric function were taken from $10^{-1}$ to $10^{7} \mathrm{~Hz}$, from -150 to $170{ }^{\circ} \mathrm{C}$.

\section{Results and Discussion}

Initial investigations with the Fourier Transform Infrared Spectroscopy (FT-IR) and Complex Dielectric Relaxation (CDR) techniques were concerned on the chemical structure of PHB before and after extrusion, in order to evaluate if this process could yield degradation products that would influence its properties, such as crystallinity and mobility of the polymeric chain. In order to characterize PHB degradation due to procedure, injected samples were also studied, in which were analyzed, by Size Exclusion Chromatography (SEC), Differential Scanning Calorimetry (DSC) and Melt Flow Index (MFI) along with all the processing cycle, modifications of molecular weights and thermal properties.

Table 1. Processing parameters used for PHB extrusion and injection.

\begin{tabular}{|c|c|c|c|c|c|c|c|c|}
\hline \multicolumn{9}{|c|}{ PHB Extrusion } \\
\hline \multirow{2}{*}{ Screw Speed (RPM) } & \multirow{2}{*}{ Flow (kg/h) } & \multicolumn{7}{|c|}{ Temperature $\left({ }^{\circ} \mathbf{C}\right)$} \\
\hline & & $\mathbf{Z 1}$ & $\mathbf{Z 2}$ & $\mathbf{Z 3}$ & $\mathbf{Z 4}$ & $\mathbf{Z 5}$ & Die & Melted \\
\hline 200 & 1.5 & 100 & 150 & 165 & 165 & 170 & 170 & 175 \\
\hline \multicolumn{9}{|c|}{ PHB Injection } \\
\hline \multicolumn{2}{|c|}{ Pressure (bar) } & \multicolumn{7}{|c|}{ Temperature $\left({ }^{\circ} \mathbf{C}\right)$} \\
\hline Final & Holding & $\mathbf{Z 1}$ & $\mathbf{Z 2}$ & $\mathbf{Z 3}$ & $\mathrm{Z4}$ & $\mathbf{Z 5}$ & & Mold \\
\hline 560 & 330 & 158 & 162 & 174 & 174 & 172 & & 30 \\
\hline
\end{tabular}




\subsection{Fourier Transform Infrared Spectroscopy (FT-IR)}

Figure 1 shows the IR spectra of PHB before and after processing by extrusion. The characteristic absorption bands of PHB can be seen at $2928 \mathrm{~cm}^{-1}$ corresponding to the methylene $\mathrm{C}-\mathrm{H}$ vibration, the symmetric $-\mathrm{C}-\mathrm{O}-\mathrm{C}-$ stretching vibration in the range of $800-975 \mathrm{~cm}^{-1}$ and the $\mathrm{C}=\mathrm{O}$ stretching at $1722 \mathrm{~cm}^{-1[16]}$. There are many studies regarding the use of FT-IR technique to determine the crystallization degree of $\mathrm{PHB}$ by monitoring the $\mathrm{C}=\mathrm{O}$ stretching vibrational region (1780-1700 $\left.\mathrm{cm}^{-1}\right)^{[17,18]}$. It is known that the most evident spectral changes during PHB crystallization are in the carbonyl band at $1740-1720 \mathrm{~cm}^{-1}$, where the amorphous phase presents a broad band near $1738 \mathrm{~cm}^{-1}$, while the crystalline phase band shifts to lower wavenumbers near $1722 \mathrm{~cm}^{-1[13]}$. Also, the asymmetric $-\mathrm{C}-\mathrm{O}-\mathrm{C}-$ stretching vibration in the range of $1180-1150 \mathrm{~cm}^{-1}$ may shift, when the degree of crystallization is reduced after the processing ${ }^{14}$. According to these findings and since no significant differences between samples before and after processings were observed in all spectra of Figure 1, it can be concluded that the extrusion conditions showed at Table 1 causes no effect in the reduction of the degree of crystallization or cause drastic chemical changes on the PHB chain.

\subsection{Complex Dielectric Relaxation (CDR)}

CDR analysis was used in order to determine glass transition temperature $\left(\mathrm{T}_{\mathrm{g}}\right)$ and possible changes on the polymer chains mobility caused by their reduction size in PHB samples. Figure 2 shows plots of the dielectric loss, $\varepsilon$ ", as a function of temperature at different frequencies for PHB before (Figure 2a) and after (Figure 2b) extrusion. Two main relaxation processes, which appear as a maximum in $\varepsilon$ " ( $\alpha$ and $\beta$, in order of decreasing temperature), were detected in the investigated temperature range. The graphs of dielectric relaxation at constant frequency (isochronal graphs) show a $\beta$-relaxation starting at $-50{ }^{\circ} \mathrm{C}$, and an $\alpha$-relaxation starting at $0{ }^{\circ} \mathrm{C}$. It is known that PHB presents these primary $\alpha$ and $\beta$ relaxations ${ }^{19,20}$, where $\beta$-relaxation was attributed to local shifts of ester groups attached to the polymeric chains, while the $\alpha$-relaxation was ascribed to shifts in the polymer chains in the amorphous phase in the range of the glass transition temperature. These relaxations also shift to higher frequencies as the temperature rises, due to the higher mobility resulting from heating. The temperature dependence of the $\beta$-process follows an Ahrrenius behavior, while the $\alpha$-process displays a curvature at the higher measured frequencies characteristic of a Vogel-Fulcher-Tamman dependence, as already observed in previous works ${ }^{19,20}$.

For semi crystalline polymers, like PHB, at temperatures higher than $\mathrm{T}_{\mathrm{g}}$, the amorphous polymer chains are confined to move between the crystalline regions. This restriction leads to an $\alpha$-relaxation behavior, well established in literature by the CDR technique. When some degradation occurs, it can result in a polymer crystallinity variation, leading to a modification in the dynamics of the $\alpha$-relaxation. If the polymer degradation improves crystallinity, its $\alpha$-relaxation will change in three aspects: a) a decrease in the intensity; b) an increase in relaxation time; and c) a concurrent change in its shape, where amorphous systems are asymmetric and the presence of crystals produce a broadening and a symmetrization of the relaxation. A comparative analysis
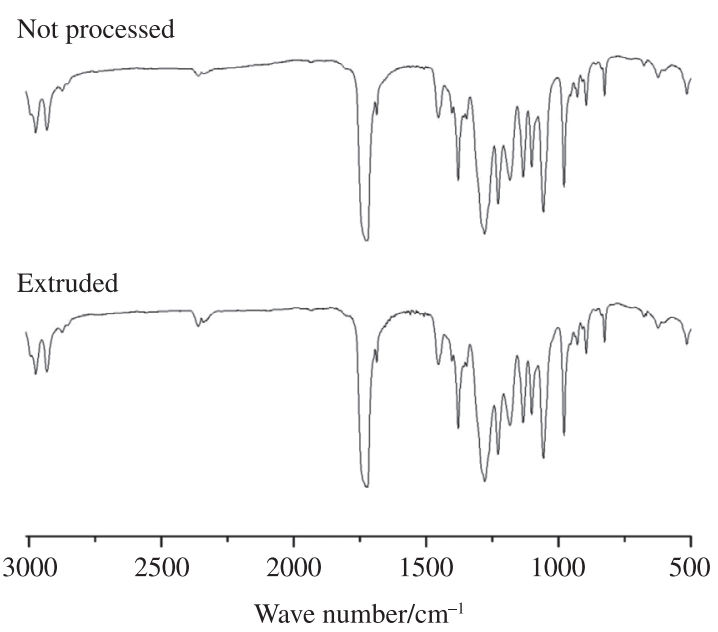

Figure 1. FT-IR spectra of PHB before and after processing by extrusion.

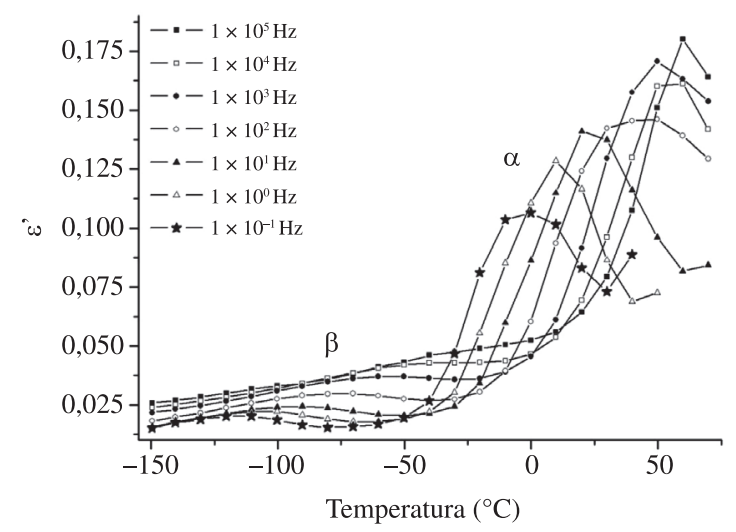

(a)

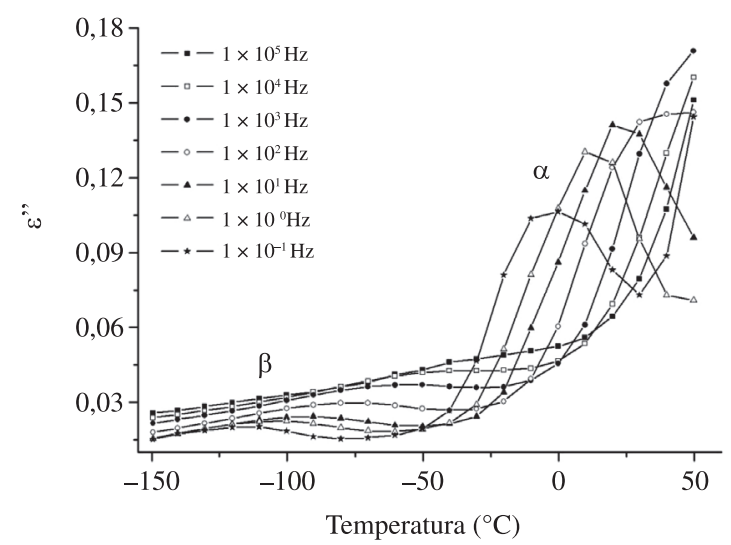

(b)

Figure 2. Isochronal dielectric loss, $\varepsilon$ ", as a function of temperature for PHB before and after processing by extrusion from $10^{-1}$ to $10^{5} \mathrm{~Hz}$. 
Table 2. Thermal transitions of PHB before and after processing measured by DSC.

\begin{tabular}{|c|c|c|c|c|c|c|c|}
\hline PHB & $T_{g}\left({ }^{\circ} \mathbf{C}\right)$ & $T_{c c}\left({ }^{\circ} \mathbf{C}\right)$ & $\Delta H_{c c}\left(\mathbf{J} \cdot \mathbf{g}^{-1}\right)$ & $T_{m}\left({ }^{\circ} \mathbf{C}\right)$ & $\Delta H_{m}\left(\mathbf{J} \cdot \mathbf{g}^{-1}\right)$ & \multicolumn{2}{|c|}{$\begin{array}{c}\text { Crystallinity } \\
\mathrm{X}_{\mathrm{c}}^{*}(\%)\end{array}$} \\
\hline \multicolumn{8}{|c|}{ Before processing } \\
\hline $1^{\text {st }}$ Cycle & - & - & - & $139 \quad 168$ & $11.6 \quad 79.7$ & 7.95 & 54.6 \\
\hline $2^{\text {nd }}$ Cycle & 0.25 & 61.5 & -69.5 & 168 & 82.7 & & \\
\hline \multicolumn{8}{|l|}{ Extruded } \\
\hline $1^{\text {st }}$ Cycle & - & - & - & 166 & 85.9 & & \\
\hline $2^{\text {nd }}$ Cycle & 1.05 & 50.3 & -39.6 & 169 & 89.0 & & \\
\hline \multicolumn{8}{|c|}{ Extruded and injected } \\
\hline $1^{\text {st }}$ Cycle & - & - & - & 171 & 82.0 & & \\
\hline $2^{\text {nd }}$ Cycle & 1.30 & 49.8 & -38.0 & 170 & 89.0 & & \\
\hline
\end{tabular}

$* \mathrm{Xc}=$ degree of crystallinity estimated from the DSC thermograms as described in Equation 1. The standard deviations $(\sigma)$ are smaller than $2{ }^{\circ} \mathrm{C}$.

of the isochronal graphs in Figure 2, however, indicates a similar behavior for PHB before and after extrusion, presenting a $\mathrm{T}_{\mathrm{g}}$ of $1.0{ }^{\circ} \mathrm{C}$ for both samples. These results show that processing did not affect the polymeric relaxation for PHB, but a further thermal analysis is needed in order to investigate crystallization processes after extrusion and injection.

\subsection{Differential Scanning Calorimetry (DSC) and Melt Flow Index (MFI)}

Figure 3 shows DSC thermograms of PHB samples in the first and second heating runs and in the cooling scan. First heating cycle shows an endothermic peak assigned as the polymer melting. The curve of no processed PHB indicates the presence of spherulites with melting temperature slight inferior than the whole material. This behavior is not seen by the processed samples since they had already suffered a heating cycle during extrusion and injection, promoting the previous melting of these small crystals and completely mixture into the polymer.

In the second heating cycle, it is possible to estimate the glass transition temperature $\left(T_{g}\right)$ as the shift near $1.0^{\circ} \mathrm{C}$ in the thermograms of all studied samples (as already verified by CDR results), while the endothermic peaks indicate the melt temperature $\left(T_{m}\right)$ near $170{ }^{\circ} \mathrm{C}$. Table 2 shows the specific values of temperature and enthalpy for all transitions for the three samples. No significant variation was observed in the glass transition and melt temperatures for PHB before and after processing. However, in the second cycle, the exothermic peak, frequently attributed to crystallization, indicates that cold crystallization temperature $\left(T_{c c}\right)$ decreased in value when PHB is processed. The crystallization enthalpy of processed PHB is also reduced due to the partial crystallization occurred in the cooling scan between the first and second heating runs. This behavior suggests that the polymer crystallization is facilitated after processing and a higher crystallization enthalpy is observed when PHB is extruded and injected.

On the other hand, the melt flow index, Table 3, increases after every processing step. The effect of rising MFI (greater fluidity) is related to the higher mobility of the polymer chains, which occurs by the chain shearing due to degradation after extrusion and injection. The reduction
Table 3. Melt Flow Index of the three PHB samples.

\begin{tabular}{cc}
\hline PHB & $\begin{array}{c}\text { Melt Flow Index } \\
(\text { g/10 minutes })\end{array}$ \\
\hline Before processing & $19 \pm 2$ \\
Extruded & $21 \pm 2$ \\
Extruded and injected & $26 \pm 2$ \\
\hline
\end{tabular}

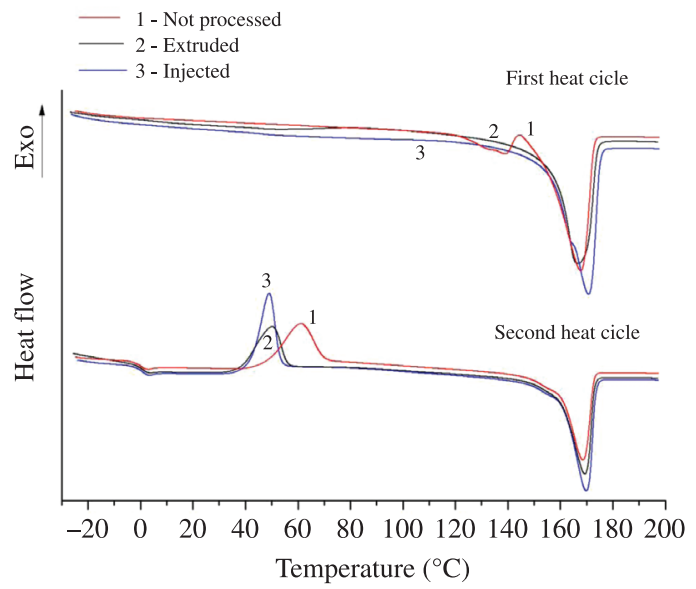

(a)

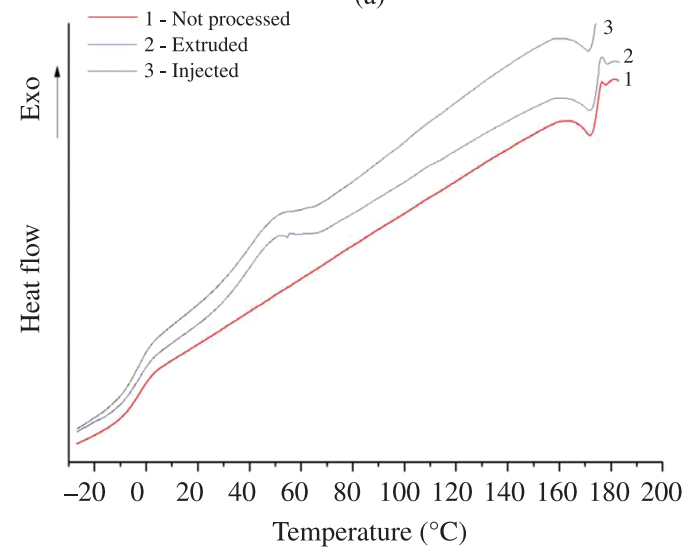

(b)

Figure 3. DSC curves showing: a) first and second heating runs, and b) cooling scan of PHB before processing, after extrusion and after extrusion followed by injection. 
Table 4. Molar mass values measured by SEC, and calculated considering only the thermal degradation effect for PHB samples before and after extrusion and injection processing.

\begin{tabular}{|c|c|c|c|c|c|}
\hline PHB & $\overline{M_{n}}\left(10^{3} \mathrm{~g} \mathrm{~mol}^{-1}\right)$ & $\overline{M_{w}}\left(10^{3}{\left.\mathrm{~g} \cdot \mathrm{mol}^{-1}\right)}^{-1}\right.$ & Polydispersity & $\frac{\overline{M_{w}}}{\overline{M_{n}}}$ & $\overline{M_{n}} *$ (theoretical) \\
\hline Before processing & 246 & 535 & 2.17 & & - \\
\hline Extruded & 175 & 383 & 2.19 & & 245 \\
\hline Injected & 104 & 208 & 1.99 & & 244 \\
\hline
\end{tabular}

*Molar mass values calculated according to Kuniok et al. ${ }^{4}$, using $k_{d}=2.2 .10^{-8} \mathrm{~min}^{-1}$ at $175^{\circ} \mathrm{C}$.

observed in $T_{c c}$ may be related to the increasing mobility of the polymeric chains, caused by the reduction in molar mass, when shearing and thermal degradation occur due to process. This interpretation is also congruent with the degradation mechanism of PHB, which is based on cis-elimination reactions and scission of the polymeric chain, lowering its molar mass ${ }^{4,5}$.

\subsection{Size Exclusion Chromatography (SEC)}

In order to corroborate the interpretation of the results showed by DSC and MFI analysis, the variation in the PHB molecular weight after each processing step was investigated by SEC. Table 4 shows the molar mass values obtained from PHB before process, and samples obtained after extrusion and injection. These results show a huge drop of PHB molar mass after each processing step, and it also observes a reduction in the polydispersity, proportional to the reduction of the molar mass, which indicates a systematic and preferential rupture of the high length chains. According to Kunioka et al. ${ }^{4}$, this chain rupture occurs by a random scission of an ester group of the structure, generating low molar mass products and forming carboxylic and crotonate vinyl groups in a rate calculated by Equation 2:

$\frac{1}{P_{N, 1}}-\frac{1}{P_{N, 0}}=k_{d} \cdot t$

where $P_{N, 0}$ is initial average molar mass, $P_{N, 1}$ is the molar mass after a specific time $t$, and $k_{d}$ is the thermal degradation rate, given by the Arrhenius relation, with an activation energy of $212 \mathrm{~kJ} \cdot \mathrm{mol}^{-1}$.

Using the residence time of PHB at extrusion and injection ( 0.5 minutes for each step) and the average molar mass of the polymer before processing $\left(245,790\right.$ g.mol $\left.{ }^{-1}\right)$ as $P_{N, 0}$, the average molar mass of the polymer after each processing step can be estimated, considering that there are losses only due to the thermal degradation at $175^{\circ} \mathrm{C}\left(k_{d}=2 \cdot 2 \cdot 10^{-8} \mathrm{~min}^{-1}\right)^{[4]}$. Results calculated by using Equation 1 are also showed in Table 4. If only thermal degradation could occur during 0.5 minutes of extrusion at $175^{\circ} \mathrm{C}$, average molar mass loss of PHB should be only $0.3 \%$ of its original value, while experimental results showed a reduction of $29 \%$. Similar conclusions can be taken when results from injected $\mathrm{PHB}$ are analyzed. It suggests that the processing of PHB causes not only thermal degradation of the polymer, but the mechanical forces involved at extrusion and injection also promote the reduction of its molar mass by the shearing of the polymeric chains.

\section{Conclusions}

The aim of this work is to understand the degradation occurred in PHB when this biodegradable polymer is processed in large scale by melt processing techniques such as extrusion and injection. Although thermal degradation of this polymer is largely studied, poor attention is paid to the effect of the mechanical forces that are also involved in the industrial processing techniques. Chemical changes in PHB after processing could not be detected by FT-IR and CDR, although evidences of some degradation were observed by the decreasing on the $\mathrm{T}_{\mathrm{cc}}$ and the increasing of MFI values after extrusion and injection. These effects are justified by the reduction of the molar mass when PHB is processed, causing the high mobility of its polymeric chains, as already predicted when thermal degradation occurs.

Molar mass data obtained by SEC for PHB before processing were used to estimate the reduction of molar mass, due to thermal degradation of the processed polymer and compared with the experimental values obtained from PHB after extrusion and after extrusion followed by injection. Results showed that the reduction of the polymeric chain after industrial processing was higher than expected, suggesting that thermal degradation was not the main cause to promote this decrease of the PHB molar mass. In the conditions studied in this work, the shearing of the polymeric chain existing in extrusion and/or injection processes acts as an important factor to considerate when dealing with large scale/industrial applications of PHB.

\section{Acknowledgements}

Authors gratefully acknowledge Dr. Tiberio A. Ezquerra, Instituto de Esctrutura de la Materia, IEM-CISIC, for CDR analysis, and to PHB Industrial S/A for PHB supply. W. M. Pachekoski acknowledges CAPES and FAPESB/CNPq for financial support. 


\section{References}

1. Sharma R and Ray A. Polyhydroxybutyrate, its copolymers and blends. Journal of Macromolecular Science - Review Macromolecular Chemical Physics. 1995; C35(2):237.

2. Chandra R and Rustgi R. Biodegradable Polymers. Progress Polymer Science. 1998; 23(7):1273-335. http://dx.doi. org/10.1016/S0079-6700(97)00039-7

3. Scott G and Gillead D. Degradable Polymers. London: Chapman \& Hall; 1995. http://dx.doi.org/10.1007/978-94011-0571-2

4. Kunioka M and Doi Y. Thermal degradation of microbial copolyesters: poly (3 -hydroxybutyrate - co - 3 - hydroxyvalerate) and poly (3 - hydroxybutyrate -co-4-hydroxybutyrate). Macromolecules. 1990;23(7):1933-6. http://dx.doi.org/10.1021/ma00209a009

5. Grassie N, Murray EJ and Holmes PA. The thermal degradation of poly(-(d)- $\beta$-hydroxybutyric acid): Part 2 - Changes in molecular weight. Polymer Degradation and Stability. 1984; 6(2):95-103. http://dx.doi.org/10.1016/0141-3910(84)90075-2

6. Asrar J and Gruys K. Biodegradable Polymer (Biopol). In: Doi Y. Polyesters III: Applications and commercial products. Weinheim: Wiley, VCH; 2004. Biopolymer vol. 4.

7. Hoffman A, Kreuzberge S and Hinrichsen G. Influence of thermal degradation on tensile strength and Young's modulus of poly(hydroxybutyrate). Polymer Bulletin. 1994; 33(3):355-9. http://dx.doi.org/10.1007/BF00314274

8. Kopinke FD, Remmler M and Mackenzie K. Thermal decomposition of biodegradable polyesters-I: Poly( $\beta$-hydroxybutyric acid). Polymer Degradation and Stability. 1996; 52(1):25-38. http://dx.doi. org/10.1016/0141-3910(95)00221-9

9. Yamagushi M and Arakawa K. Effect of thermal degradation on rheological properties for poly(3-hydroxybutyrate). European Polymer Journal. 2006; 42(7):1479-86. http://dx.doi. org/10.1016/j.eurpolymj.2006.01.022

10. D'Almeida AR and Dias ML. Comparative study of shear degradation of carboxymethylcellulose and poly(ethylene oxide) in aqueous solution. Polymer Degradation and Stability. 1997; 56(3):331-7. http://dx.doi.org/10.1016/ S0141-3910(96)00187-5

11. Renstad R, Karlsson S and Albertsson A. The influence of processing induced differences in molecular structure on the biological and non-biological degradation of poly (3-hydroxybutyrate-co-3-hydroxyvalerate), P(3-HB-co-3-HV).
Polymer Degradation and Stability. 1997; 57(3):331-8. http:// dx.doi.org/10.1016/S0141-3910(97)00028-1

12. Modi S, Koelling K and Vodovotz Y. Assessment of PHB with varying hydroxyvalerate content for potential packaging applications. European Polymer Journal. 2011; 47(2):179-86. http://dx.doi.org/10.1016/j.eurpolymj.2010.11.010

13. Liao Q, Noda I and Frank CW. Melt viscoelasticity of biodegradable poly(3-hydroxybutyrate-co-3hydroxyhexanoate) copolymers. Polymer. 2009; 50(25):6139-48. http://dx.doi. org/10.1016/j.polymer.2009.10.049

14. Hong S-G, Gau T-K and Huang S-C. Enhancement of crystallization and thermal stability of polyhydroxybutyrate by polymeric additives. Journal of Thermal Analysis and Calorimetry. 2011; 103(3):967-75. http://dx.doi.org/10.1007/ s10973-010-1180-3

15. Barham PJ, Keller A, Otun EL and Holmes PA. Crystallization and morphology of a bacterial thermoplastic: poly-3-hydroxybutyrate. Journal of Materials Science. 1984; 19(9):2781-94. http://dx.doi.org/10.1007/ BF01026954

16. Hong K, Sun S, Tian W, Chen GQ and Huang W. A rapid method for detecting bacterial polyhydroxyalkanoates in intact cells by Fourier transform infrared spectroscopy. Applied Microbiology and Biotechnology. 1999; 51(4):523-6. http:// dx.doi.org/10.1007/s002530051427

17. Kansiz M, Damínguez-Vidal A, McNaughton D and Lendl B. Fourier-transform infrared (FTIR) spectroscopy for monitoring and determining the degree of crystallization of polyhydroxyalkanoates (PHAs). Analytical and Bionalytical Chemistry. 2007; 388(5-6):1207-13. PMid:17530232. http:// dx.doi.org/10.1007/s00216-007-1337-5

18. Huang H, Guo W and Chen H. In situ FTIR and generalized 2D IR correlation spectroscopic studies on the crystallization behavior of solution-cast PHB film. Analytical and Bioanalytical Chemistry. 2011; 400(1):279-88. PMid:21336794. http:// dx.doi.org/10.1007/s00216-011-4750-8

19. Sics I, Tupureina V, Kalnins M, Ezquerra TA and Baltá-Calleja FJ. Dielectric relaxation of poly-(hydroxybutyrate) relating to microstructure. Journal of Macromolecular Science - Physics. 1998; B37(6):851.

20. Nogales A, Ezquerra TA, Garcia JM and Baltá-Calleja FJ. Structure-Dynamic relationships of $\alpha$-relaxation in flexible capolyesters during crystallization as revealed by real-time methods. Journal of Polymer Science. 1999; 37:37. 